\title{
http://artnodes.uoc.edu
}

\section{Aproximación al teatro lorquiano desde la teoría de las redes sociales: La casa de Bernarda Alba}

\author{
Elena Martínez Carro \\ Universidad Internacional de La Rioja (UNIR) \\ Fecha de presentación: marzo de 2019 \\ Fecha de aceptación: mayo de 2019 \\ Fecha de publicación: julio de 2019
}

\section{Cita recomendada}

Martínez Carro, Elena. 2019. «Aproximación al teatro lorquiano desde la teoría de las redes sociales: La casa de Bernarda Alba». Artnodes. N. ${ }^{\circ}$ 24: 134-141. UOC [Fecha de consulta: dd/mm/aa]. http://dx.doi.org/10.7238/a.v0i24.3298

$\begin{array}{cl}\text { C) } & \text { Los textos publicados en esta revista están sujetos -si no se indica lo contrario- a una licencia de } \\ \text { Reconocimiento } 4.0 \text { Internacional de CreativeCommons. La licencia completa se puede consultar en } \\ \text { https://creativecommons.org/licenses/by/4.0/deed.es_ES. }\end{array}$

\section{Resumen}

La casa de Bernarda Alba de García Lorca ha sido motivo de numerosos estudios filológicos en las últimas décadas. La última obra del poeta es incuestionable como clásico teatral. Por otra parte, las actuales investigaciones digitales, tanto la marcación textual como la aplicación de la teoría de las redes sociales a los textos dramáticos, permiten estudiar esta obra de manera cuantitativa y gráfica y realizar una comparativa con los estudiosos críticos y filológicos precedentes. A través de la marcación en lenguaje XML-TEl de la obra teatral lorquiana, y su volcado en programas como Ghepi, puede observarse la organización del universo dramático como una red social que el poeta quiso plasmar en el escenario. La constatación de la coincidencia en algunos aspectos entre los estudios tradicionales y el análisis digital y la divergencia en otros, especialmente los referidos al protagonismo de las mujeres en la obra, demuestra la existencia de nuevas vías de interpretación del teatro lorquiano, como se pretende demostrar.

\section{Palabras clave}

La casa de Bernarda Alba, teoría de las redes sociales, teatro lorquiano, marcación textual, grafos 


\title{
artnodes
}

http://artnodes.uoc.edu

Aproximación al teatro lorquiano desde la teoría...

\section{Approach to Lorca's theatrical work through Social Network Theory: The House of Bernarda Alba}

\begin{abstract}
The House of Bernarda Alba by García Lorca has been the object of numerous philological studies in recent decades. The poet's last work is undoubtedly a classic piece of theatre. Current digital research, both in textual marking as well as the application of Social Network Theory to drama texts, allows us to study this work quantitatively and graphically and to compare this research with the previous critical and philological studies. By marking Lorca's theatrical work in XML-TEI language and utilising the output from programs such as Ghepi, we can see how the dramatic universe is organised as a social network that the poet wanted to express on stage. Verifying the agreement on certain aspects between the traditional studies and digital analysis and the divergence on other aspects, especially those referring to the role of women in the work, demonstrates that there are new ways of interpreting Lorca's theatrical work as it is intended to be shown.
\end{abstract}

\section{Keywords}

The House of Bernarda Alba, Social Network Theory, Lorca's theatrical work, text marking, graphs

«Lorca es el más grande autor dramático de nuestro tiempo. [...] El teatro de Lorca no es, quizá, actual; tampoco creo que lo fuese cuando se creó, y esa es su ventaja. Pues, desde su creación es más bien la honda vigencia de un clásico la que posee» (Antonio Buero Vallejo 1960, 8).

Las relaciones sociales han sido objeto de estudio desde el nacimiento de las Humanidades. Cómo se articulan las mismas y qué repercusiones tienen en los ámbitos geográficos, históricos y artísticos han supuesto uno de los objetos principales de investigación de todos los tiempos. En las últimas décadas se ha iniciado el análisis de estas redes mediante representaciones gráficas 0 «grafos» con el fin de visualizar y analizar la estructura de una red, así como la centralidad y conectividad de cada uno de sus miembros en función de su posición (Rodríguez Treviño 2013, 104). El grafo muestra un grupo de elementos denominados nodos que están relacionados a través de correspondencias representadas por aristas. De esta forma, mediante la visualización del grafo, se muestran patrones de relación o comunidades que no son posibles de determinar si el análisis se realiza de forma individual.

Este tipo de aproximación a los datos responde al concepto de lo que Moretti (2013b) ha denominado distant reading o macroanálisis. Se presta atención a los patrones o características generales más que a los detalles de un conjunto amplio de datos.

Resulta especialmente interesante trasladar los sistemas sociales, y su representación mediante grafos, al campo de la literatura y, concretamente, a la exposición visual de las relaciones que se establecen entre los miembros de una comunidad literaria (Van Vugt 2017), 0 bien entre los personajes que protagonizan una novela (Isasi 2017).
Recientemente, Krug y Jannidis (2016), Oleza (2013) y Moretti (2013a) comentaron la versatilidad del texto dialógico y teatral para mostrar redes sociales internas dentro de una obra. Las estructuras creadas en el escenario son un reflejo de los mundos que el autor crea mediante un conflicto. A través de la visualización de las estructuras dramáticas, es posible conocer la forma compositiva del dramaturgo.

En gran medida, esto es posible gracias a la digitalización y marcación de los textos en los últimos años, que admite el análisis de los metadatos incluidos en las obras teatrales. Así, el volcado en programas gráficos permite visualizar las relaciones entre los personajes de forma precisa. Ya en Operationalizing (2013a), Moretti contabilizó el número de palabras intercambiadas entre los personajes, con la finalidad de medir el peso de los vínculos y estudiar el personaje principal para comprobar si protagonismo y centralidad coincidían. A partir de esta investigación, han sido múltiples los acercamientos a las relaciones sociales dentro de las obras literarias, tanto en prosa como en poesía, pero no cabe duda de que el mundo teatral -por ser una red social en escena- es óptimo para este tipo de estudios.

Si toda obra teatral es en sí una red social, el teatro lorquiano es posiblemente uno de los más significativos en este aspecto. Lorca intentó reflejar el mundo femenino en el que vivían las mujeres de su época. Buscó los espacios más representativos para encuadrar sus dramas y trató de expresar un mundo de sentimientos en torno a la mujer española de la primera mitad del siglo xx.

Desde esta perspectiva, puede decirse que su teatro es -posiblemente- uno de los más propicios para visibilizar no solo las redes teatrales literarias, sino las redes sociales en escena como reflejo del mundo del poeta. 


\section{artnodes}

http://artnodes.uoc.edu

El grupo GHEDI de la Universidad Internacional de La Rioja (UNIR) (Gómez et al. 2015; Jiménez et al. 2017) ha marcado en lenguaje XML-TEl algunas de las obras más significativas de Federico García Lorca como: La zapatera prodigiosa, Bodas de sangre, La casa de Bernarda Alba, Yerma y Doña Rosita la soltera, que pueden encontrase disponibles en Github.

Esta intervención sobre las obras teatrales del poeta y dramaturgo permite combinar el estudio crítico y filológico tradicional con el análisis de los datos de manera computacional, como los utilizados para las investigaciones sobre redes sociales.

A través del marcado TEl y su tratamiento en el programa Ghepi, de libre acceso, propio para el estudio de redes sociales, pueden extraerse esquemas que reflejan las relaciones entre los seres lorquianos, así como el valor cuantitativo de cada personaje dentro de la obra. Su visualización nos permite conocer de manera objetiva a los protagonistas por encima de interpretaciones hermenéuticas, así como sus principales relaciones y la transcendencia de cada una de ellas.

El programa Gephi permite no solo visualizar el esquema de la tragedia lorquiana gráficamente, sino la centralidad de grado (degree centrality) mediante el número de vínculos o conexiones que tiene cada nodo, o personaje, en la obra; la centralidad de vector propio (eigenvector centrality), que calcula la influencia por conexión de cada nodo, es decir señala a aquellos personajes que están mejor conectados en la red; y finalmente, entre otros muchos datos, la centralidad de intersección (betweenness centrality), que indica la importancia de los nodos en un gráfico basado en las trayectorias más cortas.

Aunque del análisis se derivan más datos, las tres medidas suelen determinar el protagonismo en una obra de teatro, pues el mayor número de aristas conectadas en un nodo muestran la cantidad de intervenciones de un personaje y, por tanto, sus principales conexiones y su centralidad. Estas tres medidas, en el caso de la obra Iorquiana, muestran un increíble equilibrio más propio de la poesía que del teatro en prosa. El autor granadino dejó una huella rítmica en sus tragedias, que son eminentemente poéticas, no solo por sus temáticas y lenguaje, sino por una proporción interna que puede visualizarse en los datos cuantitativos derivados del análisis digital del texto, como pretende mostrar este artículo.

\section{Las tragedias lorquianas y La casa de Bernarda Alba}

El camino hacia la tragedia de protagonismo femenino fue definido por el mismo Lorca después del estreno de Bodas de sangre en 1933:

«Ahora voy a terminar Yerma, una segunda tragedia mía. La primera fue Bodas de sangre. Yerma será la tragedia de la mujer estéril. [...] Una
Aproximación al teatro lorquiano desde la teoría...

tragedia con cuatro personajes principales y coros, como han de ser las tragedias. Hay que volver a la tragedia. Nos obliga a ello la tradición de nuestro teatro dramático. Tiempo habrá de hacer comedias, farsas. Mientras tanto yo quiero dar al teatro tragedias» (García Lorca 2017, 325-326).

El poeta escribió Bodas de sangre en 1931, Yerma en 1934 y culminó la trilogía con Doña Rosita, la soltera en 1935, estrenada el 13 de diciembre del mismo año. Las tres tragedias le llevaron a tener un gran éxito entre el público del momento, acostumbrado a un teatro comercial. Sin embargo, Lorca no vio en vida el estreno de La casa de Bernarda Alba que terminó en 1936, dos meses antes de su prematura muerte. Para entonces su triunfo como autor dramático ya se había consolidado.

El estudio de las cuatro obras ha sido tan prolijo que cualquier intento de síntesis sobrepasa los límites de este artículo, aunque conviene recordar algunos trazos generales sobre las tragedias femeninas Iorquianas con el fin de comprender -en su justa medida- la aportación cuantitativa e interpretativa digital de la más significativa de ellas.

Las cuatro tragedias están marcadas por el protagonismo femenino en una España antigua e inmovilista que somete a sus mujeres bajo un mundo asfixiante y sin salida. La España de entonces, con sus ideas arcaicas, se extiende en cada drama desde distintas perspectivas y deja entrever el mundo femenino rural que Lorca conocía muy bien desde su infancia. Mujeres que sometidas a los moldes de la época ansían el amor, la maternidad y el matrimonio como únicas formas de realización personal, al tiempo que son estigmatizadas y juzgadas por el entorno. Si bien es cierto que antes de llevar a cabo estas obras finales, Lorca quiso realizar un teatro alejado de los moldes tradicionalistas como el mismo declaró:

«Yo en el teatro he seguido una trayectoria definida. Mis primeras comedias son irrepresentables. Ahora creo que una de ellas, Así que pasen cinco años, va a ser representada por el Club Anfistora. En estas comedias imposibles está mi verdadero propósito. Pero para demostrar una personalidad y tener derecho al respeto he dado otras cosas» (García Lorca 2017, 459).

Las últimas tragedias demuestran un camino intermedio entre la innovación teatral -que el poeta había intentado en sus primeras obras- y un deseo de triunfo en las tablas del momento, tratando de mantener -en estos últimos años- una lucha por prestigiar un teatro sometido a las leyes comerciales con el fin de hacer una comunicación moral, una comunicación de denuncia (García Montero 1986, 361-362):

«No tengo ningún interés en ser antiguo o moderno, sino ser yo, natural. Sé muy bien cómo se hace el teatro semi-intelectual, pero eso no tiene importancia. En nuestra época, el poeta ha de abrirse las venas para los demás. Por eso yo, a parte de las razones que antes le decía, me he 


\section{artnodes}

http://artnodes.uoc.edu

entregado a lo dramático, que nos permite un contacto más directo con las masas» (García Lorca, 2017, 360).

Las cuatro obras tienen en común el protagonismo femenino. Después de Bodas de sangre, Lorca se desvincula de los títulos argumentativos para dejar paso a nombres simbólicos femeninos: Yerma, Rosita y Bernarda. Títulos que marcan la temática desde el inicio y que nos llevan a identificar el protagonismo con el drama que cada uno de ellos encierra. Puede afirmarse que las cuatro creaciones forman parte de una unidad temática (Josephs y Caballero 1983, 15) en torno a la mujer y el mundo en el que viven, un mundo rural en cuanto a su contextualización, pero que se eleva por encima de las barreras del tiempo y del espacio, tanto por la profundidad de sus protagonistas como por la problemática planteada en cada obra. Estas características hacen que estos dramas de Lorca perduren en el tiempo y sigan cuestionando al espectador actual los sentimientos femeninos de cualquier época y la necesaria libertad sobre los cánones establecidos.

El manuscrito de La casa de Bernarda Alba está fechado el 19 de junio de 1936 y en él Lorca había conseguido superar las contradicciones entre las que había vivido como dramaturgo entre el teatro «intelectual» y el teatro para el pueblo (García Montero 1986, 370). En la obra plantea un mundo femenino con un referente masculino, Pepe el Romano -ser ausente y presente-, que consigue plasmar un universo único de sentimientos encontrados y contradictorios, no en torno al amor, sino a una constelación de pasiones patentes en el espacio cerrado y asfixiante de la casa.

El espacio, tantas veces estudiado, determina el centro del drama (García Montero 1986, 366) y -en cierta medida- es la casa la que genera el conflicto dramático por un luto que logra un mundo cerrado; sin embargo, el dolor por la muerte del padre está ausente frente al ansiado amor. Solo un lugar hermético puede conseguir un sinfín de conflictos personales donde las pasiones se desatan y las frustraciones dan lugar a la muerte. El binomio amor-muerte está presente desde el inicio para conseguir una dramaturgia que se aboca a una única solución.

La obra comprende una síntesis de los elementos lorquianos que anteriormente el poeta había trabajado, como la experimentación, el simbolismo y la unidad temática, pero lo que sin duda hace de La casa de Bernarda Alba una obra cumbre es su concepción de teatro poético. Este concepto, en su expresión más genuina lorquiana, sorprendió en su época y fue explicitado por el dramaturgo en muchas de sus declaraciones, pues constituía una de las constantes búsquedas del poeta: «Tengo un concepto del teatro en cierta forma personal y resistente. El teatro es la poesía que se levanta del libro y se hace humana» (García Lorca 2017, 458).

Sin embargo, el público, acostumbrado a entender teatro poético como teatro en verso, necesitó de numerosas declaraciones del autor para explicitar un concepto rico y esencial en su obra:
Aproximación al teatro lorquiano desde la teoría...

«El teatro que ha perdurado siempre es el de los poetas. Siempre ha estado el teatro en manos de los poetas. $Y$ ha sido mejor el teatro en tanto era más grande el poeta. No es -claro- el poeta lírico, sino el poeta dramático. [...] La gente está acostumbrada al teatro poético en verso. Si el autor es un versificador, no ya un poeta, el público le guarda cierto respeto. Tiene respeto al verso en teatro. El verso no quiere decir poesía en el teatro [...] la obra de éxito perdurable ha sido la de un poeta, y hay mil obras escritas en versos muy bien escritos que están amortajadas en sus fosas» (García Lorca 1973, II, 983).

El teatro poético de Lorca alcanza en esta obra su máxima expresión, aunque se encargará de desnudar los diálogos de la medida del verso, de las canciones y coros a las que había acudido en otras ocasiones. Comentaban Salazar (2016), en la famosa anécdota referida a los ensayos, que Lorca cada vez que terminaba una escena exclamaba con entusiasmo: «¡Ni una gota de poesía! ¡Realidad! ¡Realismo!» (Del Río 1940, 248).

Sin embargo, y en contra de lo que pueda parecer, Lorca hizo de esta obra un verdadero poema, porque en ella consiguió un ritmo inusitado desde la prosa con una intención artística y plástica como en ninguno otro de sus dramas: «El teatro necesita que los personajes lleven un traje de poesía y al mismo tiempo que se les vean los huesos y la sangre» (García Lorca 2017, 458), «porque el suyo no es teatro poético a la manera de los modernistas, no es poesía en el teatro» (Sánchez 1950, 9), sino un teatro que tiene los elementos esenciales de la poesía, ritmo, musicalidad, equilibrio, metáfora y símbolo, todo menos medida del verso. Es decir, es poesía del teatro, no una puesta en escena de versos, sino un concepto poético de lo que es el teatro.

Posiblemente, este es uno de los elementos que se muestran de manera más significativa desde la perspectiva digital. Puede afirmarse, que después de 80 años, el último drama del poeta granadino sigue vigente y susceptible a nuevas interpretaciones y contribuciones, a pesar de los numerosos estudios que permiten observar esta obra como un clásico perdurable en el tiempo y bajo nuevas interpretaciones.

\section{Una red social en escena: La casa de Bernarda Alba}

Como señalamos al inicio de este artículo, cabe preguntarse si las nuevas perspectivas digitales pueden añadir valor a los ingentes estudios filológicos sobre las obras teatrales lorquianas y concretamente al análisis de La casa de Bernarda Alba. Cualquier obra teatral es en sí -y por esencia- un mundo de relaciones sociales llevado a la escena, donde el conflicto es imprescindible para desarrollar una trama y donde el diálogo constituye el hilo conductor. Cómo se construyen las relaciones en escena y su originalidad es una de los objetivos fundamentales para cualquier dramaturgo. 


\section{artnodes}

http://artnodes.uoc.edu

Aproximación al teatro lorquiano desde la teoría...

Los actuales estudios computacionales sobre redes sociales nos permiten acercarnos a las vinculaciones entre los personajes, así como a las relaciones que se crean en escena de manera gráfica y visual, al mismo tiempo que nos proporcionan un análisis cuantitativo del diálogo como elemento principal que constituye la obra.

Para llevar a cabo este análisis computacional, se partió de la edición publicada en 1945 por Losada en Buenos Aires. ${ }^{1}$ Cada personaje Iorquiano ha sido señalizado -a través de la marcación TEI- por las características básicas y comunes que se encuentran en toda obra teatral, como son: nombre (label), sexo (sex: $\mathrm{f} / \mathrm{m}$ ), papel en la obra (role: protagonist, antagonist, lover, other), naturaleza (nature: person, animal), importancia (importance: primary, secondary, minor). Esta sencilla señalización permite la comparación entre obras de distinta índole, debido a que responde a un común denominador existente entre todas ellas.

Lógicamente, esta marcación responde a una interpretación previa a la extracción de datos y por tanto susceptible de cambios posteriores una vez llevado a cabo el estudio cuantitativo. Si bien es cierto que en la mayoría de los casos es posible la coincidencia entre la interpretación cualitativa -que se ha desarrollado en los estudios filológicos de estos dramas- y la cuantitativa, esto no siempre es así en la obra lorquiana como mostraremos.

Como se recordará, esta obra carece de escenas, pues está dividida en tres actos. Sin embargo, atendiendo a las ocasiones en las cuales Lorca se refirió a ellas de manera explícita, y teniendo en cuenta las referencias de numerosos críticos, se han introducido en la marcación, de acuerdo con los cambios de personaje en el escenario. Esta señalización en nuestra edición ${ }^{2}$ no altera los resultados cuantitativos del programa, pero ayuda a ver el equilibrio de la obra en las entradas y salidas de los personajes a las tablas. Lorca quiso mostrar que todas las mujeres están presentes y ausentes al mismo tiempo ante el espectador en el escenario, pues o hablan entre ellas o hablan de ellas (ausentes), lo que impide ver de manera explícita la sucesión de escenas que se engarzan de manera magistral. Las escenas no están escritas, pero existen de forma plenamente organizada. ¿Cuál

Tabla 1. Elaboración propia

\begin{tabular}{|c|c|c|c|c|c|c|c|}
\hline Label & $\operatorname{Sex}$ & Roler & Importance & Nature & $\begin{array}{l}\text { Degree } \\
\text { centrality }\end{array}$ & $\begin{array}{l}\text { Betweenness } \\
\text { centrality }\end{array}$ & $\begin{array}{l}\text { Eigenvector } \\
\text { centrality }\end{array}$ \\
\hline Bernarda & $\mathrm{F}$ & protagonist & primary & person & 14 & 2,02381 & 1 \\
\hline Angustias & $\mathrm{F}$ & other & secondary & person & 14 & 2,02381 & 1 \\
\hline Magdalena & $\mathrm{F}$ & other & minor & person & 14 & 2,02381 & 1 \\
\hline Martirio & $\mathrm{F}$ & other & secondary & person & 14 & 2,02381 & 1 \\
\hline Adela & $\mathrm{F}$ & antagonist & primary & person & 14 & 2,02381 & 1 \\
\hline La Poncia & $\mathrm{F}$ & antagonist & primary & person & 14 & 2,02381 & 1 \\
\hline Criada & $\mathrm{F}$ & other & minor & person & 14 & 14,857143 & 0,965476 \\
\hline Amelia & $\mathrm{F}$ & other & minor & person & 13 & 1 & 0,959272 \\
\hline Mujer $1^{\mathrm{a}}$ & $\mathrm{F}$ & other & minor & person & 12 & 0 & 0,918619 \\
\hline Mujer $2^{\mathrm{a}}$ & $\mathrm{F}$ & other & minor & person & 12 & 0 & 0,918619 \\
\hline Mujer $3^{a}$ & $\mathrm{~F}$ & other & minor & person & 12 & 0 & 0,918619 \\
\hline Mujer $4^{\mathrm{a}}$ & $\mathrm{F}$ & other & minor & person & 12 & 0 & 0,918619 \\
\hline Muchacha & $\mathrm{F}$ & other & minor & person & 12 & 0 & 0,918619 \\
\hline$M^{\mathrm{a}}$ Josefa & $\mathrm{F}$ & other & minor & person & 7 & 0 & 0,551991 \\
\hline Prudencia & $\mathrm{F}$ & other & minor & person & 7 & 0 & 0,551297 \\
\hline Mendiga & $\mathrm{F}$ & other & minor & person & 1 & 0 & 0,077638 \\
\hline
\end{tabular}

1. La edición electrónica de la obra fue tomada de epublibre y señalizada en nuestra edición como $<$ bibl type="print-source" $>$; $<$ date $>1945</$ date $>$; $<$ publisher $>$ Losada $</$ publisher $>$; $<$ pubPlace $>$ Buenos Aires $<$ /pubPlace $>$; $<$ /bibl $>$; $<$ bibl type="electronic-source" $>$; $<$ date $>2015<$ /date $>$; $<$ publisher $>$ epublibre $<$ /publisher $>$; $<$ pubPlace $>$ Unknown $</$ pubPlace $>$; $<$ reftarget="https://www.epublibre.org/libro/detalle/1947" $></$ ref $>;</$ bibl $>;<$ bibl type="first-published" $>;<$ date $>1945</$ date $>$; $<$ publisher $>$ Losada $</$ publisher $>$; $<$ pubPlace $>$ Buenos Aires $<$ /pubPlace $>$.

2. La division de escenas figura < div cert="low" n="3" resp="\#jct" type="scene">. También aparece descrito en el revisión Desc: <change when="2017-07-27" who="\#jct">División de escenas artificiales realizada, señalada como cert low. $<$ /change $>$. 


\section{artnodes}

http://artnodes.uoc.edu

Aproximación al teatro lorquiano desde la teoría...

es la causa por la que Lorca no quiso explicitarlas? Al parecer, trató de evitar una ruptura continua en el texto de algo que era evidente y señalaba el ritmo de la obra.

Una vez llevada a cabo la marcación con los criterios mencionados, la obra tratada fue volcada al programa Gephi, desde donde pudimos extraer los metadatos cuantitativos que vemos en la tabla 1.

El análisis de los mismos nos muestra el valor de cada nodo (personaje) y sus distintas conexiones con el resto de los personajes (aristas). El primer dato es la centralidad del grado (degree centrality) de cada uno de los nodos, que «corresponde con la idea general de centralidad, donde un punto es central si está bien conectado con los demás puntos de su entorno» (Rodríguez Treviño 2013, 124). En este caso, la centralidad del grado es idéntica en las seis mujeres. A su vez, la centralidad del vector propio (eigenvector centrality) se ocupa de medir la influencia de un nodo en la red y de este análisis se derivan datos idénticos para las seis, sean o no protagonistas, según lo señalado en la marcación desde la interpretación literaria.

El segundo dato esencial se basa en la intermediación (betweeness centrality), que «determina en qué medida un punto hace de intermediario entre otros puntos por estar situado en el camino entre ellos» (Rodríguez Treviño 2013, 124). Aunque derivado de los dos datos anteriores, la intermediación entre los nodos es coincidente. Tampoco Lorca dejó de lado el profundo equilibrio entre los personajes anónimos. Las cuatro mujeres sin nombre guardan una proporcionalidad exacta entre ellas, tanto en los nodos como en los vectores.

Estas mujeres de carácter anónimo tienen mayor peso en la obra que María Josefa y Prudencia, personajes aparentemente insignificantes desde la perspectiva cuantitativa, pero elocuentes como el de María Josefa, abuela demente que comenta las verdades de sus nietas. Sus intervenciones son pocas, pero llevan nombre propio por la importancia de lo que transmiten. Podría decirse que este es uno de los puntos donde el análisis digital disiente del hermenéutico e interpretativo, pues este dato cuantificable no expresa una realidad semántica tal y como la quiso plasmar el autor, que dio nombre a esos personajes sin querer dejarlos en el anonimato.

El informe que aporta el examen cuantitativo de La casa de Bernarda Alba es sencillamente sorprendente por su perfección. El sentido poético de la obra, al que ya hicimos referencia, queda plasmado en este análisis, donde es posible ver que las seis mujeres de la familia aparecen siempre en la misma cantidad de escenas y con la misma cantidad de personas en cada una de ellas.

La concurrencia numérica de peso en cada uno de los nodos, tanto por su grado de centralidad (degree centrality), como por las conexiones entre ellas (betweeness centrality), no deja de ser insólita y justifica plenamente lo que en numerosas ocasiones la crítica ha afirmado acerca de la obra, cuando la reconocía como verdadero drama poético sin ser verso. La medida del verso está implícita en toda ella, pero no obstaculiza una comprensión más directa y moderna del tema a través de una prosa poética de indudable interés.

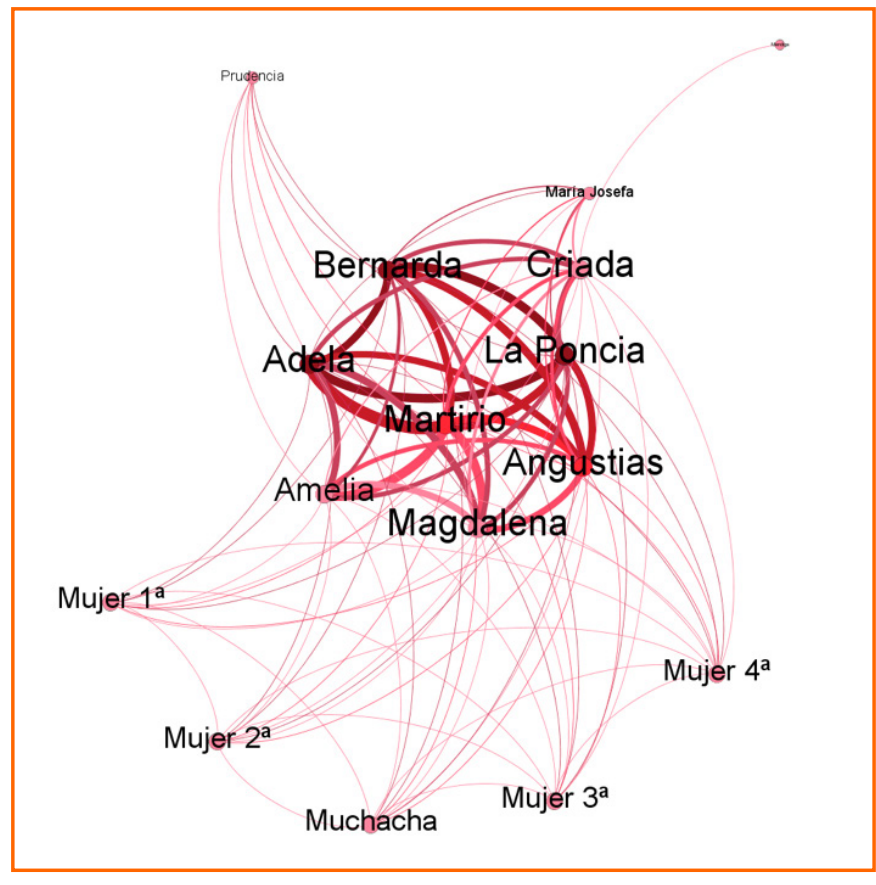

Figura 1. Elaboración propia

Estos datos y su representación gráfica llevan a entender la necesidad que tuvo el poeta de pulir constantemente esta tragedia hasta conseguir una perfección artística, como puede observarse más claramente en el grafo resultante en la figura 1.

El grafo, que llama especialmente la atención por su perfecta organización y equilibrio, tanto de los personajes principales como de los menores, muestra la disposición de las aristas y los nodos de manera circular que reflejan el mundo cerrado, en el cual se desenvuelven las mujeres de la casa. Protagonismo y espacio clausurado parecen darse la mano de manera gráfica en el análisis de sus redes.

El estudio de La casa de Bernada Alba, desde la digitalización, nos permite plantearnos distintas cuestiones sobre el concepto de protagonismo tan discutido en esta obra. ¿Es realmente Bernarda el nodo central en esta obra, tal y como señala el título? ¿Soporta su protagonismo el mayor número de conexiones? ¿Coincide realmente el protagonismo significativo -0 semántico- con el número de intervenciones de cada una de las mujeres?

Como puede observarse en la marcación inicial -y desde una perspectiva tradicional- se señaló a Bernarda como única protagonista frente a las principales antagonistas, Adela y Poncia, y al resto de las hermanas también marcados como «otros». Sin embargo, el análisis cuantitativo nos muestra que las seis mujeres son protagonistas de manera igualitaria y todas forman un círculo equilibrado y cerrado como la propia casa. Son las relaciones entre ellas las que generan un espacio verdaderamente asfixiante, como demuestra el grafo. Esta representación deja patente que no es solo la casa la que genera el conflicto, sino el complejo número de relaciones entre todas las protagonistas. Como afirmaba García Montero (1986, 366-367): 


\section{artnodes}

http://artnodes.uoc.edu

«Estamos ya ante la verdadera macrofísica del poder que se diluye, que se deshace en una red cada vez más espesa, no identificable en un solo lugar, organizado en mil hilos que pasan por cada uno de los espacios, por cada uno de los protagonistas, cosiéndolos en un tejido común. Porque es de esto de lo que habla el texto de La casa de Bernarda Alba: no hay un lugar limitado para el poder; la historia, relegada aparentemente al campo de lo público, no respeta los pretendidos refugios de la intimidad entra y actúa dentro de ellos, los constituye, porque pertenece a su propio discurso».

Podría decirse que Lorca ha construido dos protagonismos paralelos. Uno, el que nos da a través de la fuerza de Bernanda, autoritaria e impositiva, que podríamos llamar significativo, al que se dirige el argumento a través de las relaciones con las antagonistas y que nos llevan a conducir la mirada solo hacia ella y lo que representa. Y otro, organizado a través del diálogo entre todas las mujeres, creando una red única y equilibrada que hace que todas ellas tengan un protagonismo compartido en torno a la casa. Es este el protagonismo que se da desde el análisis digital -y que el propio Lorca trabajó cuidadosamente, como si del cañazo de un tapiz se tratara-, difícil de ver inicialmente, pero sustento de toda la obra.

Si toda obra de Lorca es poética por su lenguaje, simbolismo y medida, La casa de Bernarda Alba lo será por su estudiado equilibrio en todos los ámbitos teatrales: número de personajes en escena, prosa medida y símbolos. En esta obra Lorca no utilizará canciones ni coros, tan introducidos en sus tragedias anteriores, y lo hará para dejar constancia de la posibilidad de llegar más allá de la poesía formal del verso y la canción, para dejar que sea la prosa teatral la que cante a través de las mujeres anónimas.

En definitiva, no puede afirmarse que las perspectivas filológicas y digitales sobre la obra coincidan plenamente en este tipo de análisis, pues como se ha podido ver, los personajes ausentes -como Pepe el Romano u otros con menor número de palabras- son inexistentes en la digitalización y, sin embargo, fundamentales en el argumento de la obra. El poeta cuidó especialmente el equilibrio de las relaciones en escena para que ninguna de las protagonistas quedara fuera del argumento, al mismo tiempo que dejó a los personajes menores alrededor del nudo orgánico. Son las mujeres sin nombre, que de manera anónima cierran más el círculo y lo subrayan a través de una mera comparsa. Ellas son el contacto con el exterior, como ya Lorca había hecho en Doña Rosita la soltera, que sustituyen a los coros y canciones a los que nos tenía acostumbrados el poeta en otras de sus obras.

Queda patente que ambos análisis son complementarios y que el estudio digital nos permite visibilizar un entramado de manera cuantitativa que tan solo se podía intuir desde la crítica filológica. El análisis cuantitativo viene a confirmar que una gran obra como esta soporta cualquier investigación -por pormenorizada que sea-y deja al descubierto la genialidad de un clásico, a pesar de que su temática pueda parecer alejada de los moldes sociales actuales.
Aproximación al teatro lorquiano desde la teoría...

Aunque seguramente Lorca no contabilizó las palabras de cada uno de los personajes, sí fue consciente de la genialidad de su obra:

«Federico llevaba constantemente en su bolsillo el original de La casa de Bernarda Alba. Decía que, al terminar su drama, había tenido una congoja de llanto. Creía comenzar ahora su verdadera carrera de poeta dramático» (Hernández, 1981, p. 38).

\section{Referencias bibliográficas}

Buero Vallejo, A. 1960. «Una encuesta de Ínsula: El teatro de García Lorca». Ínsula, XV, CLXVIII, 8.

García Lorca, F. 1973. Obras completas. Madrid: Aguilar.

García Lorca, F. 2017. Palabra de Lorca. Declaraciones y entrevistas completas. Barcelona: Malpaso.

García Montero, L. 1986. «El teatro, la casa y Bernarda Alba». Cuadernos Hispanoamericanos: Homenaje a García Lorca, 1: 359-370. www.cervantesvirtual.com/obra/el-teatro-la-casa-y-bernardaalba.

Gómez, S.; J. Calvo Tello; J M. González y R. Vilches. 2015. «Hacia una biblioteca electrónica textual del teatro en español de 1868-1936 (BETTE)». Texto Digital, 11(2): 171-84. https://doi. org/10.5007/1807-9288.2015v11n2p171

Hernández, M. 1981. La casa de Bernarda Alba de Federico García Lorca. Madrid: Alianza Editorial.

Isasi, J. 2017. «Acercamiento al análisis del sistema de los personajes en la narrativa escrita en español: el caso de Zumalacárregui y Mendizábal de Pérez Galdós». Caracteres. Estudios culturales y críticos de la esfera digital 6 (2): 107-137.

Jiménez, C.; E. Martínez Carro; Mª T. Santa María et al. 2017. «BETTE: Biblioteca Electrónica Textual del Teatro en Español de la Edad de Plata». Sociedad, Políticas, Saberes. Málaga: HDH: 88-89. hdh2017.es/wp-content/uploads/2017/10/Actas-HDH2017.pdf. Josephs, A. y J. Caballero. 1983. La casa de Bernarda Alba de Federico García Lorca. Madrid: Cátedra.

Krug, M.; F. Puppe; F. Jannidis; et al. 2016. «Comparison of Methods for the Identification of Main Characters in German Novels». DH2016: 578-82.

Moretti, F. 2013a. «Operationalizing: or, the function of measurement in modern literary theory». The New Left Review, 84: 103-119.

Moretti, F. 2013b. Distant Reading. Londres: Verso.

Oleza Simón, J. 2013. Biblioteca Digital Arte Lope. Valencia: Universitat de València. https://artelope.uv.es/biblioteca/

Del Río, Á. 1940. «Federico García Lorca (1899-1936)». Revista Hispánica Moderna, Vl: 3-4.

Rodríguez Treviño, J. C. 2013. «Cómo utilizar el Análisis de Redes Sociales para temas de historia». Signos Históricos, 29: 102-141. Salazar Rincón, J. 2006. «Tema y símbolo en La casa de Bernarda 


\section{artnodes}

http://artnodes.uoc.edu

Aproximación al teatro lorquiano desde la teoría...

Alba». Pirineos. Revista de la Consejería de Educación de la Embajada de España en Andorra 2: 13-17. www.researchgate.net/ publication/270104802.

Sánchez, R. G. 1950. García Lorca: estudio sobre su teatro. Madrid: Jura.
Van Vugt, I. 2017. «Using Multi-Layared Networks to Disclose Books in the Republic of Letters». Journal of Historical Network Research, 1: $25-51$.

\section{CV}

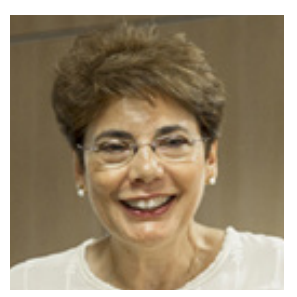

\section{Elena Martínez Carro}

Universidad Internacional de La Rioja (UNIR)

elena.martinez@unir.net

UNIR

Rectorado - Avda. de la Paz, 137, Logroño, La Rioja

Delegación Madrid - C/ Almansa, 101, Madrid

Doctora en Filología Española por la Universidad Complutense de Madrid, actualmente Decana de la Facultad de Educación de la Universidad Internacional de La Rioja (UNIR) y profesora de Didáctica de la Lengua Española y Literatura Moderna.

Desde hace años combina la actividad didáctica con la investigación en Teatro del Siglo de Oro y las Humanidades Digitales. Especializada en los dramaturgos menores del siglo xvII, ha publicado diversos artículos y libros con aportaciones documentales sobre escritores del ciclo calderoniano con el fin de acercar sus vidas y sus obras al lector actual.

En los últimos años ha centrado gran parte de su investigación en la interpretación digital de las obras de los dramaturgos de la Edad de Plata, especialmente de García Lorca.

En la actualidad pertenece a los grupos de investigación PROTEO de la Universidad de Burgos, dedicado a la edición de los textos de Agustín Moreto; GHEDI de la Universidad Internacional de la Rioja, sobre edición de textos digitales y a la Plataforma digital para la investigación y divulgación del teatro contemporáneo en Madrid, TEAMAD de la Universidad Complutense de Madrid. 